\title{
Unique Modulation of Cadherin Expression Pattern during Posterior Frontal Cranial Suture Development and Closure
}

\author{
David E. Sahar ${ }^{\mathrm{a}}$ Björn Behr ${ }^{\mathrm{a}}$ Kenton D. Fong ${ }^{\mathrm{a}} \quad$ Michael T. Longaker $^{\mathrm{a}}$ \\ Natalina Quarto ${ }^{a, b}$ \\ a Department of Surgery, Hagey Laboratory for Pediatric Regenerative Medicine, School of Medicine, \\ Stanford, Calif., USA; ${ }^{\mathrm{b}}$ Department of Structural and Functional Biology, University of Naples Federico II \\ Complesso M. S. Angelo, Naples, Italy
}

\section{Key Words}

Cranial Suture $\cdot$ Closure $\cdot$ E-cadherin $\cdot N$-cadherin .

Development $\cdot$ FGF- $2 \cdot$ Mouse

\begin{abstract}
Cranial suture development involves coordinated expression of multiple genes and tissue contribution from neural crest cells and paraxial mesoderm for timely sutural morphogenesis. Transcription factors, growth factors, and neural crest determinant genes play critical roles in calvarial growth ensuring normal development of the underlying brain. In vitro studies have implicated cell-cell adhesion molecules as a driving force behind suture closure. We performed cDNA microarray to study differential expression of adhesion molecules during the timing of suture closure in a mouse model where only the posterior frontal (PF) suture closes. Our results indicate increased expression of E-cadherin during the period of PF suture closure. Quantitative RT-PCR analysis of $E$ - and $N$-cadherin in PF closing suture revealed a biphasic expression of $\mathrm{N}$-cadherin, the first phase coinciding with cellular condensation preceding chondrogenesis followed by a second phase coinciding with E-cadherin co-expression and suture closure. Furthermore, expression analysis of the $\mathrm{N}$-cadherin and E-cadherin transcriptional repressors Wnt7a and Snail indicate a specific
\end{abstract}

temporal regulation of these genes, suggesting their potential role as regulators of both $\mathrm{E}$ - and $\mathrm{N}$-cadherin during the PF suture development and closure. Finally, given the in vitro evidence of fibroblast growth factor (FGF)-2 as a potential regulator of $E$ - and $\mathrm{N}$-cadherin we investigated the expression of E-cadherin during PF suture closure in Fgf-2 deficient mice. In contrast to in vitro data previously reported, E-cadherin expression is normal in these animals, and PF suture closure occurs properly, probably due to potential redundancy of FGF ligands ensuring normal temporal expression of $E$-cadherin and PF suture closure.

Copyright $\odot 2009$ S. Karger AG, Basel

\section{KARGER}

() 2009 S. Karger AG, Basel

Fax +41613061234 E-Mail karger@karger.ch www.karger.com www.karger.com/cto

\begin{tabular}{ll}
\hline Abbreviations used in this paper \\
\hline FGF & fibroblast growth factor \\
FGFR & fibroblast growth factor receptor \\
PF & posterior frontal \\
qRT-PCR & quantitative reverse transcriptase polymerase \\
SAG & sagittal \\
SAM & significance analysis of microarray \\
SMA & Stanford microarray database \\
SSC & saline sodium citrate
\end{tabular}

Dr. Natalina Quarto

Department of Surgery

Stanford University School of Medicine

257 Campus Drive, Stanford, CA 94305-5148 (USA)

Tel. +1 650736 1704, Fax +1 650736 1705, E-Mail quarto@ unina.it 


\section{Introduction}

Cranial suture development is a complex process involving controlled regulation of multiple genes. Several genes, including transcription factors, growth factors, and their cognate receptors are implicated in this process [Wilkie, 1997; Opperman, 2000; Rice et al., 2003]. Dysregulation at any control level would potentially lead to premature suture fusion or craniosynostosis [Wilkie, 1997]. Mutations in several genes have been implicated in pathologic development of cranial sutures, including fibroblast growth factor receptors (FGFRs), transcription factors MSX2 and TWIST, and fibrillin-1 (FBN1) [Bellus et al., 1996; Howard et al., 1997; Meyers et al., 1996; Muenke et al., 1994; Sood et al., 1996]. All of the above genes have been linked to osteoblast differentiation biology. How exactly mutations in these genes cause premature fusion of calvarial sutures has been a subject of intense study in the past decade. The overall picture of suture biology at the molecular level has become clearer in recent years using candidate gene approaches and microarray technology on cell cultures, organ cultures and in vivo sutures. Although growth and transcription factors have been clearly implicated in normal suture development, their downstream structural protein targets have not received much attention in this context.

The posterior frontal (PF) suture is of neural crest origin [Jiang et al., 2002], and is the only cranial suture which in mice closes during the second week of life [Sahar et al., 2005], while all other cranial sutures remain patent throughout life. The PF suture is endowed with a sophisticated architecture comprised of 2 layers of bone: the ectocranial and endocranial layer [Sahar et al., 2005]. We have previously demonstrated that the endocranial layer of PF suture closes through an endochondral ossification process, and Sox9 gene is a key regulator in this process [Sahar et al., 2005].

Given the sophisticated PF suture architecture and previous in vitro studies demonstrating the importance of cell-cell adhesion molecules in chondrogenesis and osteogenesis, we hypothesized that the PF closure would involve cell-cell adhesion molecules. In our initial search for candidate genes of structural proteins involved in suture patterning and closing, we performed a comparative cDNA microarray analysis between mouse PF and sagittal (SAG) calvarial sutures to investigate differential gene expression in closing versus patent sutures, respectively. Our results indicated that among the genes encoding for cell-cell adhesion molecules that were differentially up-regulated in closing sutures, E-cadherin is one of the most prominent.
Cadherins are calcium dependent cell-cell adhesion molecules that give cells the ability to create form in aggregate [Yagi and Takeichi, 2000]. These adhesion molecules are involved in tissue differentiation and morphogenesis [Gumbiner, 1996; Vleminckx and Kemler, 1999]. Cell differentiation and tissue organization require a specific repertoire of cadherins that provide cues for cell specification and commitment to a certain lineage. The failure of $E$-cadherin null mice to form epithelia or a blastocyst cavity demonstrates its pivotal role in basic morphogenetic development [Larue et al., 1994]. Moreover, differential expression of cadherin family members is responsible for cell sorting, tissue differentiation and organogenesis during development [Yagi and Takeichi, 2000; Jamora et al., 2003]. In addition, it has been demonstrated that $\mathrm{N}$-cadherin and $\mathrm{OB}$-cadherin expression is important in osteoblast biology in vitro [Okazaki et al., 1994] and in vivo [Kawaguchi et al., 2001]. Classic cadherins are a potential effector downstream of the Fgf-2/ $M s \times 2$ pathway. Both $N$ - and $E$-cadherin are expressed in normal human calvarial pre-osteoblasts and osteoblasts [Lemonnier et al., 1998]. Studies in cells harboring FGFR2 S252W gain-of-function mutation, a cause of syndromic form of craniosynostosis in humans, have shown increased cell aggregation and up-regulation of E-cadherin [Lemonnier et al., 2001].

Here we investigate the temporal-spatial expression of $E$-cadherin and $\mathrm{N}$-cadherin during the first month of life in CD-1 mice. Our results demonstrated that E-cadherin and $\mathrm{N}$-cadherin gene expression is tightly regulated during PF suture patterning and closure. Specifically, E-cadherin expression is up-regulated during the timing of $\mathrm{PF}$ suture closure, while the $N$-cadherin gene expression profile is biphasic, with a first up-regulation phase preceding chondrogenesis, followed by a second up-regulation phase coinciding with the period of PF suture closure, as well as E-cadherin co-expression. Furthermore, gene expression analysis of the $\mathrm{N}$-cadherin and E-cadherin transcriptional repressors Wnt7a and Snail [Tufan and Tuan, 2001; Batlle et al., 2000; Cano et al., 2000; Hajra et al., 2002] indicate a specific temporal regulation of these genes, suggesting their potential role as regulators of both $E$ - and $\mathrm{N}$-cadherin in the context of PF suture development and closure.

Finally, given the in vitro evidence of FGF-2 as a potential regulator of $E$-cadherin, we analyzed $E$-cadherin expression in PF suture of Fgf-2 null mice. Our results demonstrate normal E-cadherin expression and lack of abnormal phenotype in PF suture of $F g f-2$ deficient mice. 


\section{Materials and Methods}

\section{Animals}

The animals were cared for in accordance with the guidelines of the institutional animal care and the use committee at Stanford University. CD-1 mice obtained from Charles River Laboratories (Wilmington, Mass., USA) were kept in a temperature- and moisture-controlled animal facility. Animals were sacrificed on a specific post-natal (p) day that was based on birth date (day 0). Time points included: p1, p3, p5, p6, p7, p8, p9, p10, p11, p12, p13, p14, p15, p16, p17 and p25. Mice heterozygous for the Fgf2 $2^{\text {tm1Doe }}$ targeted-mutant allele were obtained from Jackson Laboratories (www.jax.org). The mouse strain of Fgf-2 null is C57BL/6J. Homozygous mice were selected by Mendelian inheritance and genotyping was performed according to Jackson Laboratories' protocol (stock number 003256).

\section{Tissue Harvest}

Mice were sacrificed by $\mathrm{CO}_{2}$ asphyxiation and the calvariae were dissected. PF and SAG sutures were meticulously harvested from CD-1 mice using Surgical Acuity ${ }^{\mathrm{TM}}$ loupes. After clearly identifying the lambdoidal, SAG, coronal, PF and anterior frontal sutures, the PF and SAG suture complexes inclusive of the suture mesenchyme, adjacent osteogenic fronts of the opposing calvarial growth plates, and the underlying dura mater were carefully dissected, separated and harvested using fine, slightly curved sharp tip scissors (www.finescience.com). Suture width after harvest measured $0.6-0.8 \mathrm{~mm}$. The length of sutures depended on animal age. The bregma was excluded. The sutures were snap frozen for RNA extraction and stored at $-80^{\circ} \mathrm{C}$.

\section{Tissue RNA Extraction}

One litter (on average 8 animals) of CD-1 mice or Fgf-2 null mice was used for each time point. Pooled sutures for each condition and time point were homogenized in $0.6 \mathrm{ml}$ of Trizol (Invitrogen, Carlsbad, Calif., USA) using a Pellet Pestle Motor (Kontes). RNA was extracted and precipitated using chloroform and 2-propanol. RNA was further washed with DEPC treated 70\% ethanol according to the manufacturer's protocol.

\section{Tissue Processing and Staining}

Following sacrifice of animals described above, the calvariae were harvested and immediately fixed in fresh, chilled $4 \%$ paraformaldehyde overnight at $4^{\circ} \mathrm{C}$ followed by $24-48 \mathrm{~h}$ of decalcification in Formical-2000 (Decal Chemical Company, Congers, N.Y., USA). The calvaria was divided coronally through the bregma to separate the PF from the SAG sutures. The specimen then underwent dehydration in a graded ethanol series and were paraffin embedded. The entire blocks were serially sectioned (5-7 $\mu \mathrm{m})$ totaling to approximately 400 sections for each block. Every other slide was stained with routine hematoxylin and eosin for evaluation and the remaining slides were used for immunohistochemistry. The stained sutures were examined with Carl Zeiss Axioplan 2 (Zeiss, Thornwood, N.Y., USA) microscope. Images were captured by AxioVision (Zeiss) and combined by Adobe Photoshop (Adobe Systems, San Jose, Calif., USA). Fusion was defined as mature bony bridge between the osteogenic fronts.

\section{cDNA Arrays}

We utilized Mus musculus cDNA large-scale microarrays manufactured by the Stanford Functional Genomics Facility (www.microarray.org). Prior to use, slides were rehydrated in a hydration chamber for $20 \mathrm{~min}$ at room temperature followed by snap drying at $7^{\circ} \mathrm{C}$. The poly-L-lysine surface was blocked by treatment with $0.05 \%$ succinic anhydride prepared in a buffer solution consisting of $350 \mathrm{ml}$ of 1-methyl-2-pyrrolidinone and $15 \mathrm{ml}$ of boric acid for $20 \mathrm{~min}$ on a shaker. The cDNAs on the slides were then denatured in distilled water for $2 \mathrm{~min}$ at $95^{\circ} \mathrm{C}$ followed by fixation with $95 \%$ ethanol. Forty micrograms of RNA per time point from PF and SAG sutures were reverse transcribed to fluorescently-labeled cDNA probes for hybrization using Cy3-dUTP and Cy5-dUTP (Amersham, Piscataway, N.J., USA). Our common reference point was RNA extracted from calvariae at several time points. After probe hybridization, the arrays were incubated overnight at $65^{\circ} \mathrm{C}$ followed by serial washing in saline sodium citrate solution. Arrays were immediately scanned using a GenePix Scanner (Axon Instruments, Foster City, Calif., USA). The array images obtained were analyzed by GenePix Pro software (Axon Instruments) and imported to the Stanford Microarray Database for further comparison and interpretation. In addition, we utilized Significance Analysis of Microarrays for statistical analysis of arrays.

\section{Quantitative Real-Time PCR}

Purified and quantified RNA was treated with DNAse I (Ambion, Austin, Tex., USA) to clear genomic DNA. Five $\mu \mathrm{g}$ of total RNA from each time point was reverse transcribed to cDNA using random primer hexamers (Invitrogen). Primers were designed with Primer Express ${ }^{\mathrm{TM}}$ software (Applied Biosystems). Each primer was subjected to RT-PCR to ensure single primary amplicon as evidenced by $2 \%$ agarose gel electrophoresis to be between 100-150 bp. Primer sequences used: E-cadherin F: GCCAATCCTGATGAAATTGGAA;R:CAGAACCACTGCCCTCGTAATC, $N$-cadherin F: CCGTGAATGGGCAGATCACT; R: TAGGCGGGATTCCATTGTCA, Snail F: ACCCACTCGGATGTGAAGAGA; R: GGAAGATGCCAGCGAGGAT, Wnt7a F: CCGTTGGAACTGCTCAGCG; R: CCGCAGCGATAATCGCATAG.

Amplified fragments were ligated into pGEM Easy Vector (Promega, Madison, Wisc., USA) and sequence was confirmed using DNA sequence analysis (Stanford Genome Technology Center, Stanford, Calif., USA). Samples along with primers and Syber Green Master Mix (Applied Biosystems) were loaded in a 384-well sealed plate and the reaction was run in ABI Prism 7900 HT (Applied Biosystems) according to the manufacturer's protocol. Gapdh (Applied Biosystems) was used for internal control. The standard curve method of quantitation was used to calculate expression of target genes relative to the housekeeping gene Gap$d h$. Four serial dilutions of cDNA (1:4) were made for the calibration curve and trend lines were drawn using $\mathrm{Ct}$ values versus log of dilutions for each target gene and Gapdh run in triplicate with correlation coefficient $\left(\mathrm{R}^{2}>0.99\right)$. Relative expressions were calculated using line equations derived from calibration curves and obtaining ratios of target gene to Gapdh for each time point. For each gene, experiments were run at least 3 times using 3 independent litters of mice. The error bars reflect variability between litters. 


\section{Statistical Analysis}

The results are presented as the mean \pm SE of 3 independent experiments. Statistical differences between the means are examined by Student's test or 1-way ANOVA between groups with Tukey's multiple comparison test on Prism software (Prism version 3.0 for Windows; GraphPad, San Diego, Calif., USA). A p value $<0.05$ was considered statistically significant.

\section{Immunohistochemistry}

Selected paraffin embedded sections were chosen and heat antigen retrieval was performed. Vectastain ABC system (Vector Laboratories, Burlingame, Calif., USA) was used according to the manufacturer's protocol. Primary antibody against E-cadherin (H-108) and N-cadherin (H-63; Santa Cruz Biotechnology, Santa Cruz, Calif., USA), were used in 1:50 to 1:500 dilutions. Biotinylated secondary antibody was used in 1:200 dilutions. Controls for each primary antibody consisted of probing with normal (irrelevant) rabbit IgG (sc-2027; Santa Cruz Biotechnology), followed by incubation with biotinylated secondary antibody. Immunostaining with the irrelevant IgG did not detect any staining (data not shown).

\section{Results}

\section{cDNA Microarray Analysis of PF and SAG Suture on} p5 and $p 15$

To search for candidate cell-cell adhesion molecules differentially expressed in PF suture during its closure, we performed cDNA microarray on PF and SAG sutures on days p5 and p15, before and after the period of suture closure. Our results revealed up-regulation (approximately 9-fold) of E-cadherin gene expression in the PF suture at day p15 compared to p5 (fig. 1a). In contrast, the expression of $E$-cadherin remained steady in SAG suture during the same time points. In order to validate the data obtained from microarray analysis, we performed quantitative real-time PCR (qRT-PCR). As shown in figure $1 \mathrm{~b}$, qRT-PCR validates the microarray results. Thus, these results indicated that E-cadherin is differentially expressed in patent versus closed PF suture.

\section{E-cadherin Expression Pattern during PF Suture \\ Development and Closure}

Next, to investigate the potential relationship between E-cadherin expression and PF suture biology, we analyzed the expression of this gene during the first month of life in the PF suture, using qRT-PCR. As shown in figure $2 \mathrm{a}$, an increase in E-cadherin expression is noted as early as day p9. Maximal expression is observed on day p15 with down-regulation by day p17. This period coincides with the period of PF suture closure, which starts around p10 and mostly completes by p15 [Sahar et al.,

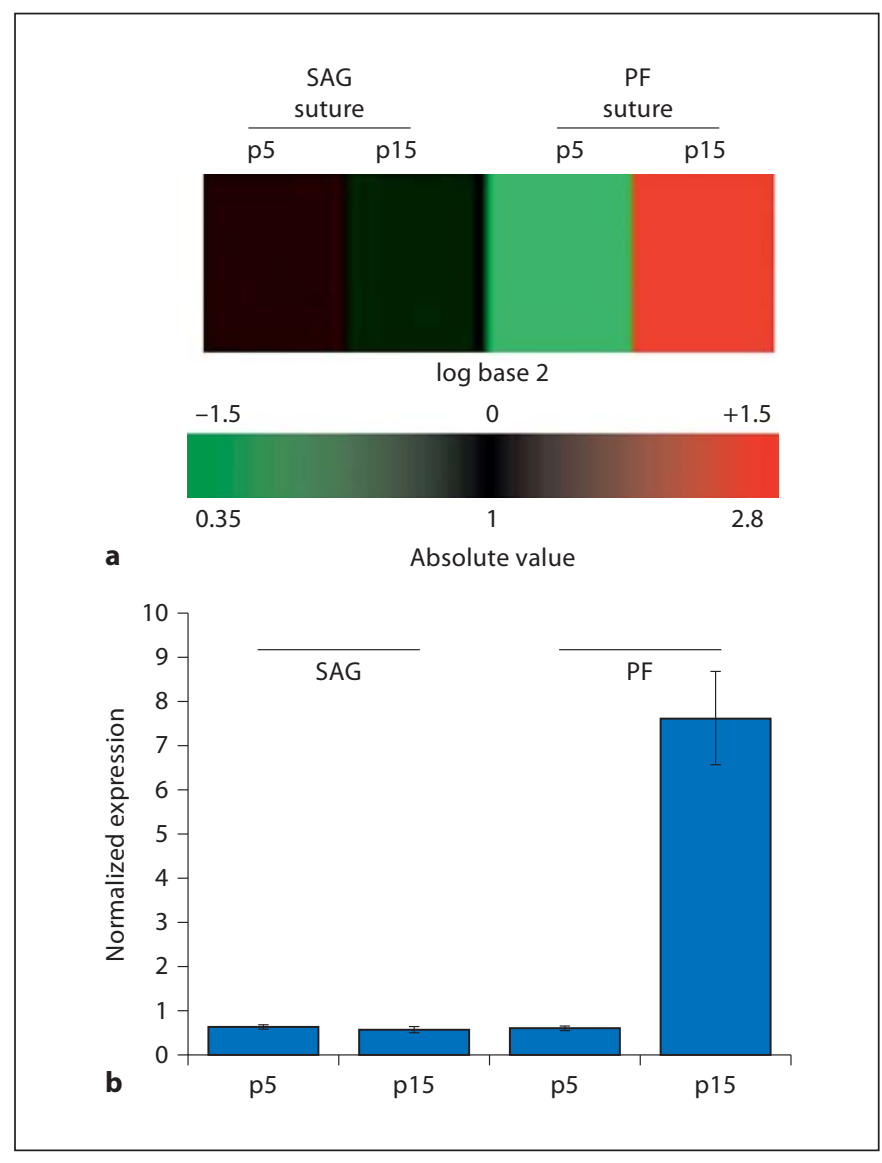

Fig. 1. Differential E-cadherin expression in cranial sutures. a Microarray profile of E-cadherin gene on SAG and PF suture shows that the gene is up-regulated exclusively in the PF suture at p15. Green $=$ Lower than mean expression; red $=$ higher than mean expression. b Quantitative real-time RT-PCR confirmed the upregulation of E-cadherin gene expression in PF suture at p15. Quantified mRNA values were normalized by the amounts of Gapdh mRNA, and results are given as fold induction. The results are presented as the mean $\pm \mathrm{SD}$ of 3 independent experiments.

2005]. To define the precise spatial localization of E-cadherin protein we performed immunohistochemistry on $\mathrm{PF}$ suture sections obtained from different time points from the beginning to the end of suture closure time. As shown in figure $2 \mathrm{~b}$, immunohistochemical analysis performed using an E-cadherin-specific antibody detected at days p5 and p7 E-cadherin positive cells mainly at the osteogenic front, in the pericranium and dura mater, while very few positive cells were present in the suture mesenchyme. At day $\mathrm{p} 9$ some chondrocytes and pre-hypertrophic chondrocytes present in the suture mesenchyme stain positively. More positive E-cadherins cells were observed in the differentiating suture mesenchyme 
at days $\mathrm{p} 9$ and $\mathrm{p} 11$. Finally, at day p15 a strong E-cadherin staining was observed in the ossified endocranial layer of $\mathrm{PF}$ suture, overlying pericranium and underlying dura mater. In addition, immunostaining was also observed in the area of suture populated by red blood cells, vessels and lymphocytes. Thus, the immunohistological data confirm the gene expression profile. Moreover, the expression profile of the E-cadherin gene on SAG suture during the first month of life did not reveal any specific up-regulation of this gene compared to that observed in PF suture (fig. 2c).

The tightly regulated expression of the E-cadherin gene suggests a potential role of this cell-cell adhesion molecule in PF suture closure.

Biphasic Expression Pattern of N-cadherin during PF Suture Development and Closure

PF suture closure occurs by endochondral ossification of endocranial layer [Sahar et al., 2005]. Given the role of $\mathrm{N}$-cadherin in mesenchymal condensation and osteoblast differentiation [Oberlender and Tuan, 1994; Ferrari et al., 2000; DeLise and Tuan, 2002; Tuan, 2003; Tuli et al., 2003; Kii et al., 2004; Stains et al., 2005], we performed qRT-PCR to examine the expression pattern of $\mathrm{N}$-cadherin during PF suture closure (fig. 3a, top panel). Interestingly, the profile of $\mathrm{N}$-cadherin appeared biphasic. The first peak expression was observed on day p7, a period when mesenchymal cell condensation is noted in the endocranial plate of the PF suture [Sahar et al., 2005]. While, the second peak was detected at days p13-p15, a period marked by osteogenesis and closure of PF suture [Sahar et al., 2005], as well as by up-regulation of E-cadherin expression (fig. 2a, b). Of interest, the expression profile of the $\mathrm{N}$-cadherin gene on SAG suture during the first month of life did not reveal any specific up- and/or downregulation of this gene, its expression profile was steady (fig. 3a, bottom panel). The expression of $\mathrm{N}$-cadherin gene in PF suture was further confirmed by immunostaining performed with specific $\mathrm{N}$-cadherin antibody (fig. 3b). At days $\mathrm{p} 5$ and p7, N-cadherin immunostaining was detected in cells present in the suture mesenchyme of the endocranial layer of PF suture, as well as at osteogenic fronts and in the pericranium. By day $\mathrm{p} 9$ very faint staining was observed in the PF suture, only the few chondroblasts present in the suture mesenchyme stained positively, while chondrocytes were negative. At day p11 $\mathrm{N}$-cadherin positive cells were completely absent in the differentiating suture mesenchyme. $\mathrm{N}$-cadherin protein was detected later in the endocranial layer of PF suture, at p13 and p15 when the PF suture closes. Immunohisto- chemistry performed with irrelevant IgG did not detect any staining (data not shown). Thus, immunohistochemistry analysis mirrored the gene expression profile. The peculiar expression pattern may reflect a dual role for $\mathrm{N}$ cadherin during PF suture closure: during cellular condensation preceding PF suture chondrogenesis, and later during osteogenesis when the PF suture closes.

\section{Expression of $\mathrm{N}$ - and $\mathrm{E}$-cadherin Transcriptional Repressors Wnt7a and Snail}

Temporally and spatially localized expression of $\mathrm{N}$ and E-cadherin in the PF suture during the period of suture closure suggests strict regulation of these 2 molecules. Previous in vitro studies have shown pre-chondrogenesis condensation events preceding overt chondrogenesis to be associated with Wnt7a-mediated $\mathrm{N}$-cadherin regulation [Tufan and Tuan, 2001; Tufan et al., 2002; Tuli et al., 2003]. Particularly, it has been demonstrated that Wnt7a mis-expression negatively affects $\mathrm{N}$-cadherin expression during limb mesenchymal chondrogenesis [Tufan and Tuan, 2001]. Given the unique and tight expression pattern of $N$ - and $E$-cadherin during the first month of life, we investigated the expression pattern of their transcriptional regulators Wnt7a and Snail, during the same period. Interestingly, Wnt7a was up-regulated in the PF suture during pre-chondrogenesis, as well as during chondrogenesis (p7-p11) and its expression returned to baseline during the period of osteogenesis (p13-p15). More important, the maximal up-regulation of Wnt7a coincided with the maximal down-regulation of $N$-cadherin. (fig. $4 \mathrm{a}, \mathrm{b}$ ) This pattern of expression would suggest that Wnt7a is a potential mediator of $\mathrm{N}$ cadherin expression in PF suture and thus, cellular condensation, is a prerequisite to differentiation along the chondrogenic pathway.

Similarly, an important transcriptional repressor of E-cadherin is the transcription factor Snail [Batlle et al., 2000; Cano et al., 2000]. Given the highly regulated expression of E-cadherin during the period of PF suture patterning and closure, we analyzed the expression of Snail during the first month of life. As shown in figure $4 c$, an up-regulated expression of Snail was noted only during the period of pre-chondrogenesis (p5-p8), marking the mesenchymal condensation period. Snail expression returned to a basal level during suture closure and osteogenesis, a period marked by up-regulation of $E$-cadherin (fig. 4d). The above expression patterns suggest that Wnt7a and Snail may be potential regulators of $E$ - and $\mathrm{N}$-cadherin expression during the PF suture patterning and closure. 


\section{E-cadherin Expression Pattern in Fgf-2 Knockout \\ Mice}

Several studies have demonstrated that in vitro FGF-2 induces expression of E-cadherin [El-Hariry et al., 2001; Debiais et al., 2001; Strutz et al., 2002; Marie et al., 2002]. Furthermore, the activating FGFR-2 mutation also causes up-regulation of E-cadherin expression in vitro [Lemonnier et al., 1998]. We have previously showed that Fgf-2 is up-regulated during PF suture development and closure. Specifically, the expression of $F g f-2$ increases at day p9 [Sahar et al., 2005]. Thus, up-regulation of $F g f-2$ expression followed by up-regulation of both, $E$ - and $N$-cadherin, may suggest a causal role. Therefore, this observation prompted us to analyze the expression pattern of E-cadherin in PF suture of Fgf-2 null mice. qRT-PCR analysis indicated that $E$-cadherin expression is not down-regulated in PF suture of $F g f-2$ null mice (fig. $5 \mathrm{a}$ ), as would be predicted based on previous in vitro data. Indeed, E-cadherin expression is similar to that observed in wild-type animals (fig. 2a). A possible explanation for the apparent contradiction between these in vivo data and in vitro data previously reported could be the redundancy of FGF ligands in the PF suture context. For instance, FGF-18, which is also expressed in the PF suture [Sahar et al., 2005] may compensate for the lack of FGF-2 ligand.

Interestingly, in Fgf-2 null mice the up-regulation of E-cadherin expression occurs earlier than in wild-type mice (fig. 2a). The trend of this expression profile has been consistently observed at least in three independent experiments. However, we cannot rule out that the earlier up-regulation of E-cadherin expression observed in the PF suture of Fgf-2 null mice is merely due to the different genetic background existing between wild-type and Fgf-2 null mice. Finally, histological analysis of PF suture did not show abnormal phenotype, but closure occurred approximately 2 days earlier than in CD-1 mice (data not shown). Furthermore, analysis of $\mathrm{N}$-cadherin did not reveal any differences in the expression pattern between Fgf-2 null and wild-type CD-1 mice (fig. 5b).

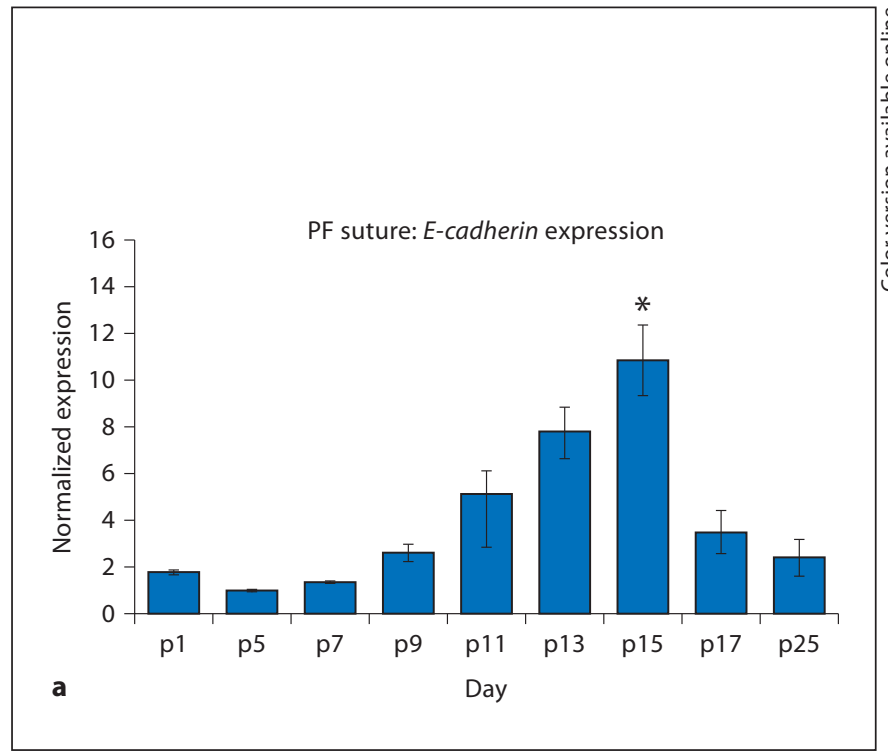

Fig. 2. a E-cadherin expression in posterior frontal suture during the first month of life. Quantitative real-time PCR for E-cadherin on $\mathrm{PF}$ suture reveals up-regulation of E-cadherin gene starting at day $\mathrm{p} 9$ with maximal expression at days p13-p15, a period coinciding with active bone deposition in the PF suture and suture closure. Quantified mRNA values were normalized by the amounts of Gapdh mRNA, and results are given as fold induction. The results are presented as the mean \pm SD of 3 independent experiments from 3 independent litters. ${ }^{*} \mathrm{p}<0.05$.

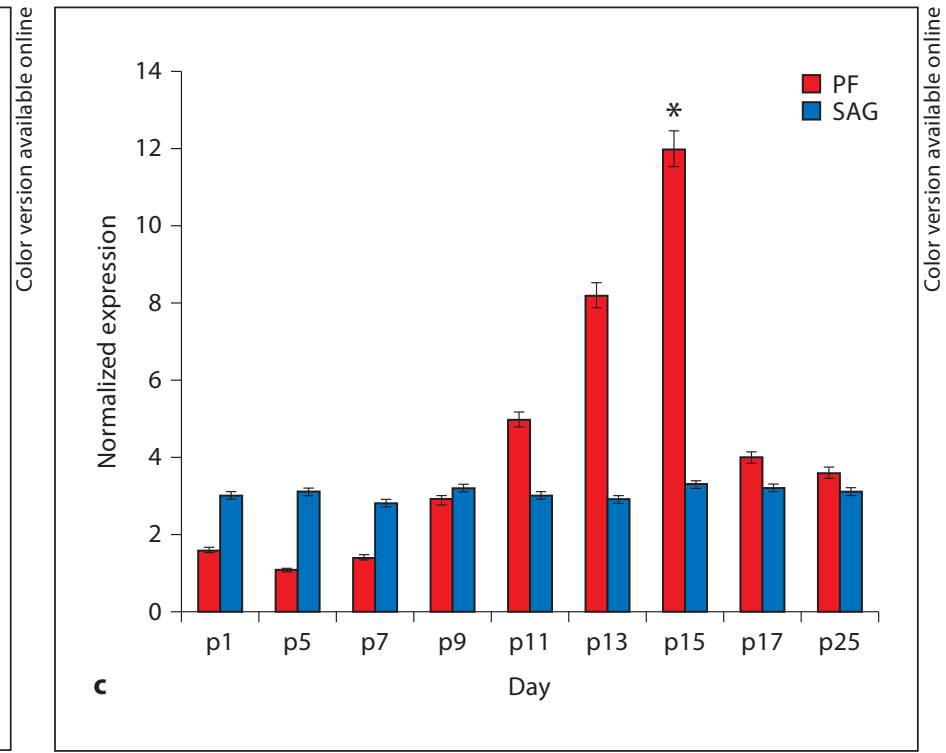

Fig. 2. c E-cadherin expression in posterior frontal suture during the first month of life. Quantitative real-time PCR for E-cadherin performed on SAG suture and its comparison with expression profile observed in PF suture. Expression of E-cadherin gene in SAG suture remains steady during the first month of life. The results are presented as the mean \pm SD of 3 independent experiments from 3 independent litters. 
Fig. 2. b E-cadherin expression in posterior frontal suture during the first month of life. Immunohistochemistry analysis of E-cadherin protein performed using a specific E-cadherin antibody, shows at day p5 absence of positive cells in the suture mesenchyme PF suture (boxed area), while positive cells are detected in the surrounding osteogenic fronts. At day p7, a faint E-cadherin staining of few cells is observed, E-cadherin staining is also detected in pericranium and dura mater cells. Starting from day $\mathrm{p}$, more E-cadherin positive cells are detected in differentiating mesenchyme of the endocranial layer of PF suture. At day p15 an intense E-cadherin staining is observed in the bony bridge of closed endocranial layer of PF suture (boxed area). Boxed areas corresponding to the suture mesenchyme undergoing to endochondral ossification are magnified in panels on the right column. Dashed lines mark the osteogenic front of approaching bone plates Note, immunostaining is also observed in the area of suture populated by red blood cells, vessels and lymphocytes (asterisk). Pr = Pericranium; $\mathrm{DM}=$ dura mater; $\mathrm{PF}-$ endo $=\mathrm{PF}$ endocranial suture. Scale bar: $200 \mu \mathrm{m}($ at $\times 20)$ and $50 \mu \mathrm{m}$ (at $\times 40)$.

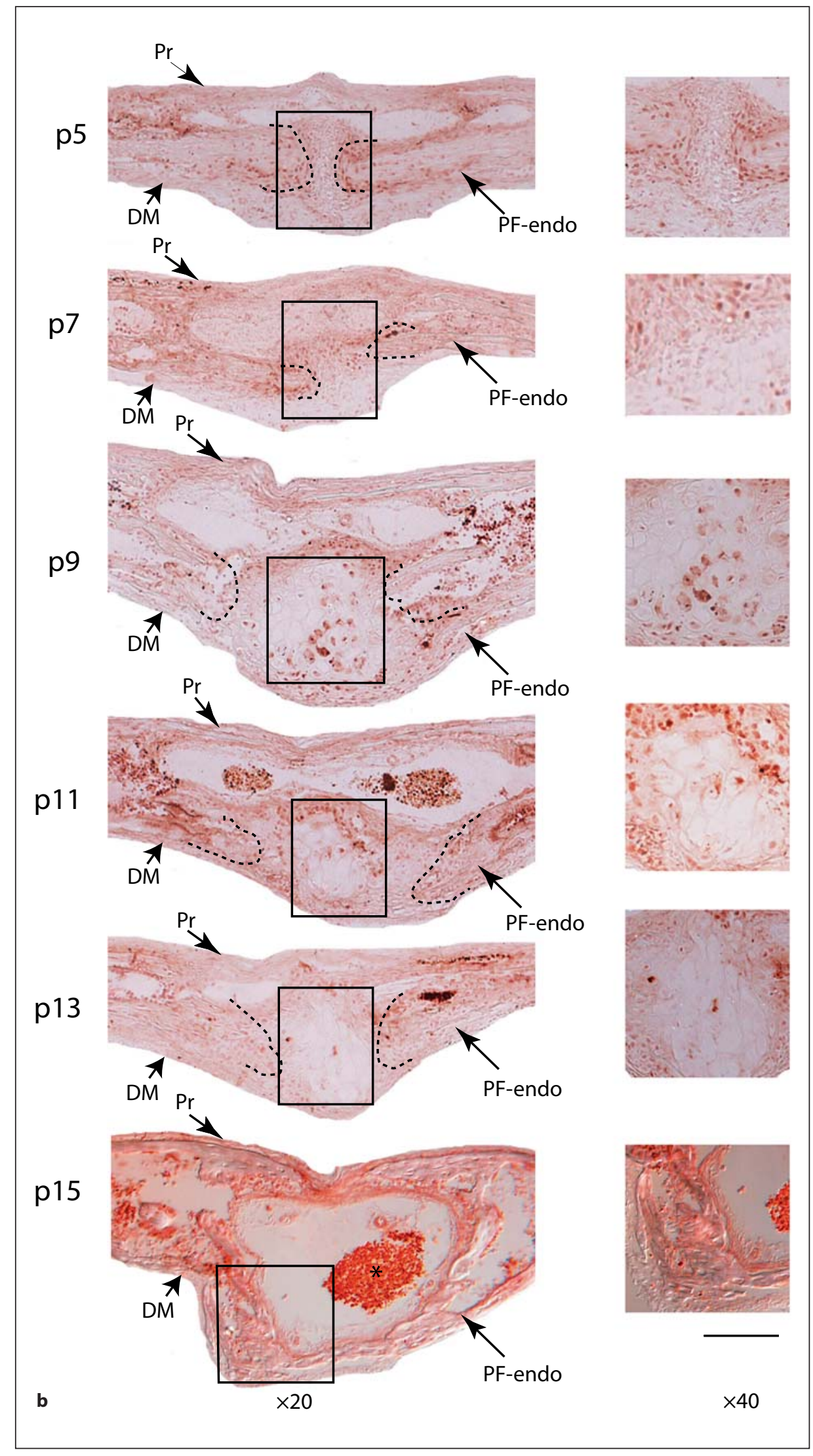




\section{Discussion}

The anatomy of PF suture is characterized by a complex architecture, and the molecular control of PF suture closure is a highly coordinated process characterized by a unique endochondral ossification of the endocranial layer [Sahar et al., 2005]. Sox9, the master regulator of chondrogenesis, is one of the genes controlling this process. To get more insights into the molecular mechanism controlling the PF suture we have performed a cDNA microarray analysis on mouse PF and SAG calvarial sutures from time points before and after closure to investigate differential gene expression in closing and patent sutures. The expression study reported here provides a basis for future functional studies with the aim to identify other genes controlling PF suture closure.

Adhesive interactions are essential not only in embryonic development, but also in a variety of other biological processes, including the differentiation and maintenance of tissue architecture. Cell-cell adhesion plays a critical role in the organization of cells to tissues during embryogenesis as well as adult life. Cadherins are a family of cell surface glycoproteins that function in promoting $\mathrm{Ca}^{2+}$ dependent cell-cell adhesion and serve as the transmembrane components of adherens junctions. The cadherins are a major class of membrane proteins with prominent roles in cell adhesion, and the regulation of tissue organization and morphogenesis.

Our interest focuses on cell-cell adhesion molecules NCadherin and E-cadherin since these molecules are regulators of chondrogenesis and osteogenesis, the 2 major events controlling the PF suture patterning and closure.

In order to differentiate along the chondrogenic pathway mesenchymal cells tightly gather together, a process known as mesenchymal condensation. This process results from active cell movement that causes an increase in mesenchymal cell packing density. $\mathrm{N}$-cadherin is one of the cell-adhesion molecules implicated in this process. The unique biphasic expression pattern of $\mathrm{N}$-cadherin in PF suture supports a potential role of this cell-cell adhesion molecule during PF patterning and closure. Our data indicate that $\mathrm{N}$-cadherin expression is temporally up-regulated during the time coinciding with the mesenchymal condensation, while a dramatic down-regulation is observed during the period of chondrogenesis followed by a second up-regulation at the end of chondrogenic differentiation when endochondral ossification and, therefore, closure of PF suture has occurred. These data strongly suggest that $\mathrm{N}$-cadherin may be involved in early events of PF suture patterning and closure, such as mesenchy- mal condensation preceding cartilage formation, and later during osteogenesis when the final step of PF suture occurs, while E-cadherin is mainly involved during the late steps of PF suture closure when the bony tissue replaces the cartilage (fig. 6). Our hypothesis is strongly supported by previous studies. Evidence indicating the involvement of $\mathrm{N}$-cadherin mediated cell-cell adhesion in cellular condensation and chondrogenesis comes from studies designed to perturb N-cadherin function. A pre-

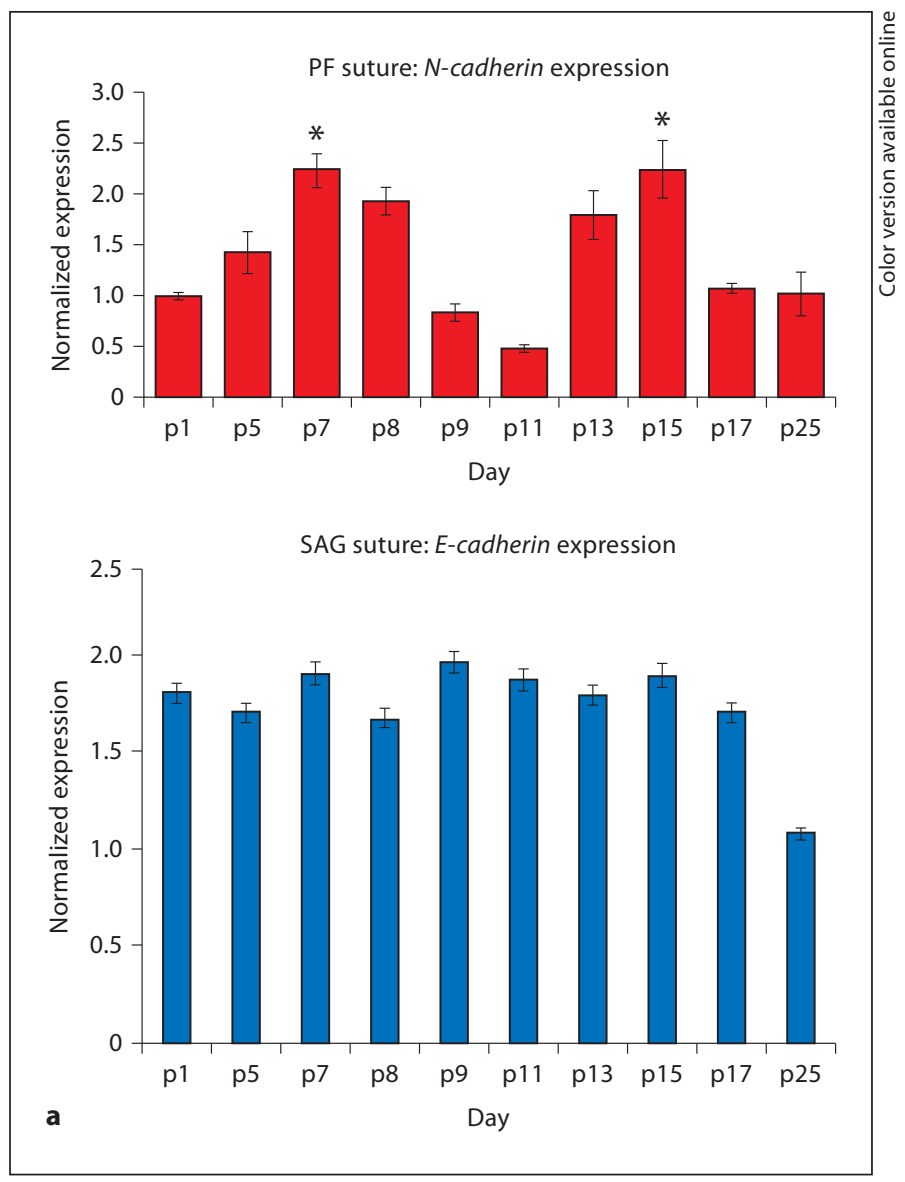

Fig. 3. a $N$-cadherin expression in the posterior frontal suture during the first month of life. Top panel: Quantitative real-time PCR for $N$-cadherin on PF suture reveals a biphasic expression profile of $N$-cadherin gene. $N$-cadherin is first up-regulated on $\mathrm{p} 7$, a period coinciding with a cellular condensation in the endocranial plate of the PF suture. A second phase of $\mathrm{N}$-cadherin gene up-regulation is observed on p13-p15, a period marked by osteogenesis and closure of PF suture. Bottom panel: Quantitative realtime PCR for E-cadherin on SAG suture shows that the expression is steady overtime. Quantified mRNA values were normalized by the amounts of Gapdh mRNA, and results are given as fold induction. The results are presented as the mean \pm SD of 3 independent experiments from 3 independent litters. ${ }^{*} \mathrm{p}<0.05$. 
Fig. 3. b $N$-cadherin expression in the posterior frontal suture during the first month of life. Immunolocalization of $\mathrm{N}$-cadherin protein on PF suture using a specific anti-N-cadherin antibody mirrors the biphasic gene expression profile and localizes $\mathrm{N}$-cadherin protein in the suture mesenchyme of the endocranial layer of PF suture during cellular precondensation at day $\mathrm{p} 5$ and p7. By day p9 only a few condroblasts present in the differentiating suture mesenchyme stain positively. At day p11 none of the cells present in the suture stain positive. In contrast, by day p13 a strong $\mathrm{N}$-cadherin staining is observed in the endocranial layer of $\mathrm{PF}$ suture. Day p15, which marks the closure of PF suture, is also characterized by strong $\mathrm{N}$-cadherin staining in the area of endochondral ossification (boxed area). Pericranium, osteoblasts and dura mater, also stain positively for $\mathrm{N}$-cadherin. Boxed areas are magnified in panels on the right column. $\mathrm{Pr}=$ pericranium; $\mathrm{DM}=$ dura mater; $\mathrm{PF}$ endo $=\mathrm{PF}$ endocranial suture. Scale bar: $200 \mu \mathrm{m}($ at $\times 20), 50 \mu \mathrm{m}$ (at $\times 40)$.

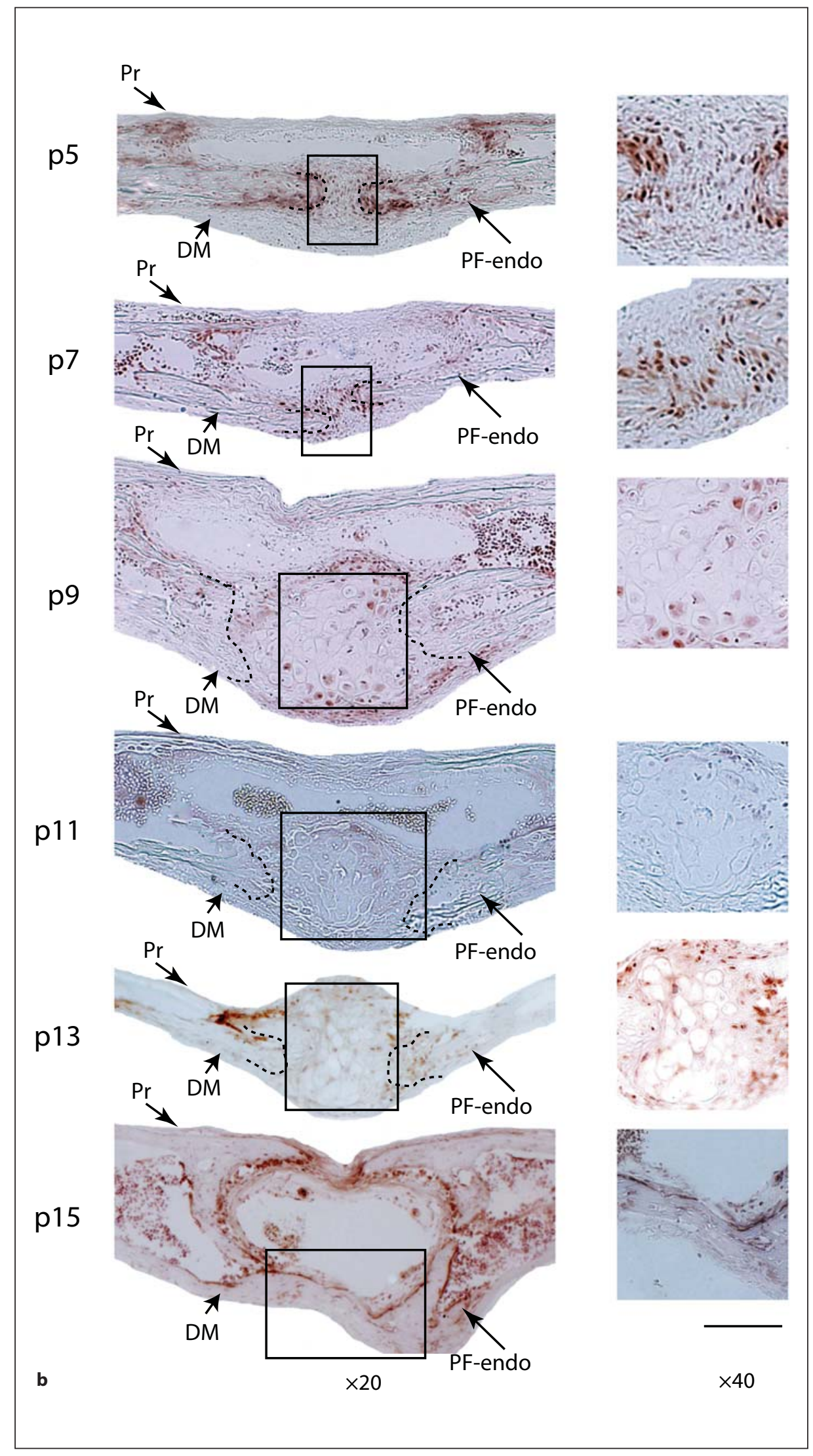



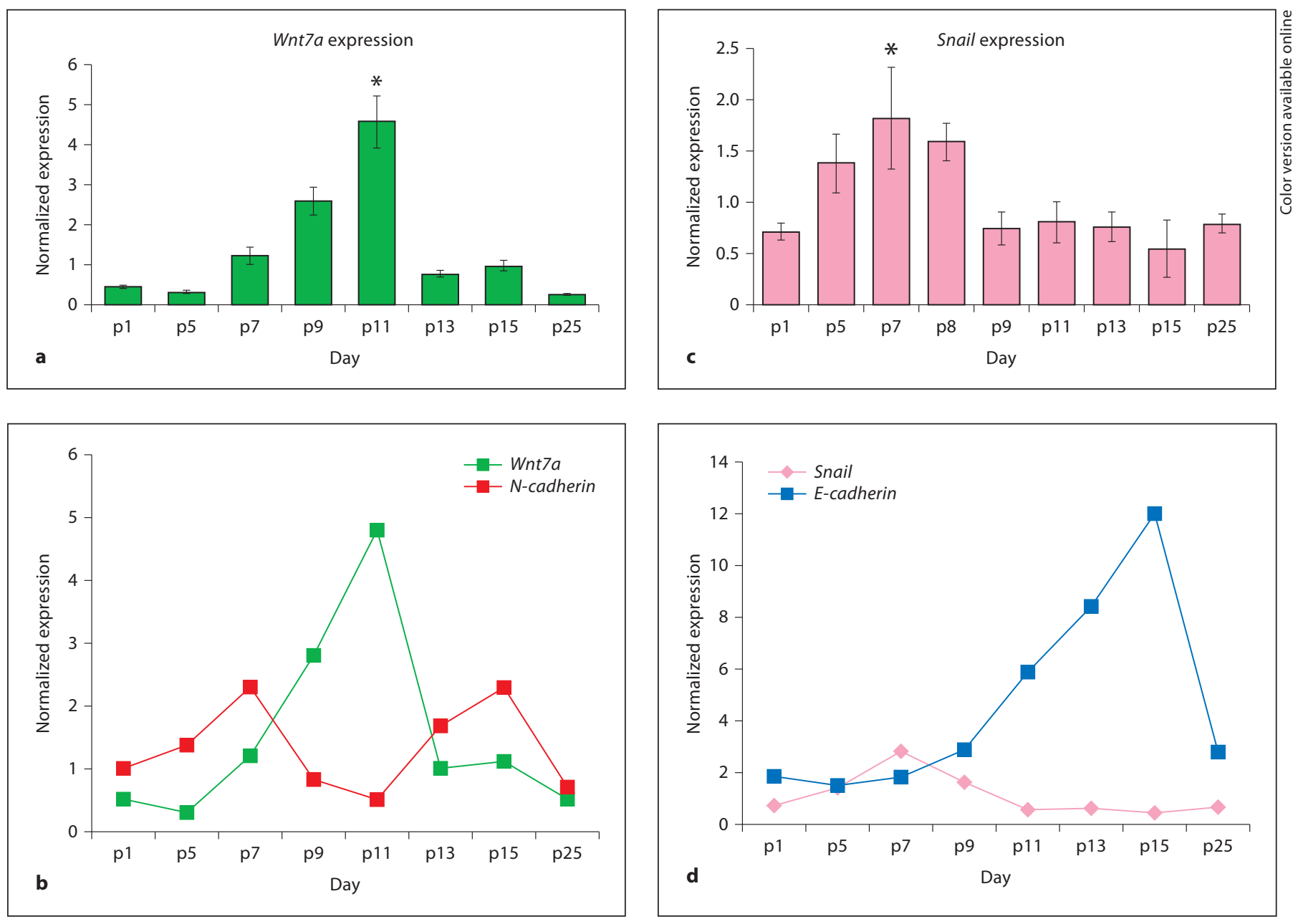

Fig. 4. Expression of $N$ - and $E$-cadherin transcriptional repressors Wnt7a and Snail. a Quantitative real-time PCR for Wnt7a on PF suture reveals up-regulation of $W n t 7 a$ gene as early as $\mathrm{p} 7$, with maximal expression on $\mathrm{p} 11$ and return to baseline by p13. b The inverse gene expression correlation between Wnt7a and $\mathrm{N}$-cadherin. Note that Wnt7a gene expression is specifically up-regulated during the down-regulation of $N$-cadherin gene. c Quantitative real-time PCR for Snail on PF suture reveals a moderate up-

vious study demonstrated a substantial inhibition of cellular condensation and chondrogenesis both in vivo and in vitro using a function-blocking monoclonal antibody directed against $\mathrm{N}$-cadherin [Oberlender and Tuan, 1994]. Moreover, it has been demonstrated that functional $\mathrm{N}$-cadherin is required in a temporally and quantitatively specific manner for normal cellular condensation and chondrogenesis to occur. Interestingly, chondrogenesis is also suppressed by over-expression of either wildtype or mutant forms of $\mathrm{N}$-cadherins, suggesting that temporally inappropriate cell-adhesion activities are in

regulation of Snail gene starting on $\mathrm{p} 5$, with a decrease on $\mathrm{p} 9$ and a return to baseline by $\left.15 .{ }^{*} \mathrm{p}<0.01\right)$. $\mathbf{d}$ Graph showing the inverse gene expression profile of Snail and E-cadherin during the PF suture patterning and closure. Quantified mRNA values were normalized by the amounts of Gapdh mRNA, and results are given as fold induction. The results are presented as the mean \pm SD of 3 independent experiments. ${ }^{*} \mathrm{p}<0.05$.

fact inhibitory to chondrogenic differentiation, possibly because of inappropriate timing of signaling events. In vitro work demonstrated that $\mathrm{N}$-cadherin is required for mesenchymal cell specification in early phases of embryonic limb bud development, and perturbation of cadherin-mediated interactions disrupts chondrogenic cell condensation [Haas and Tuan, 1999; Tuan, 2003; Tuli et al., 2003]. However, persistence of $\mathrm{N}$-cadherin expression prevents further progression from precartilage condensation to chondrocyte development, probably by stabilizing cell-cell adhesion [DeLise and Tuan, 2002]. 


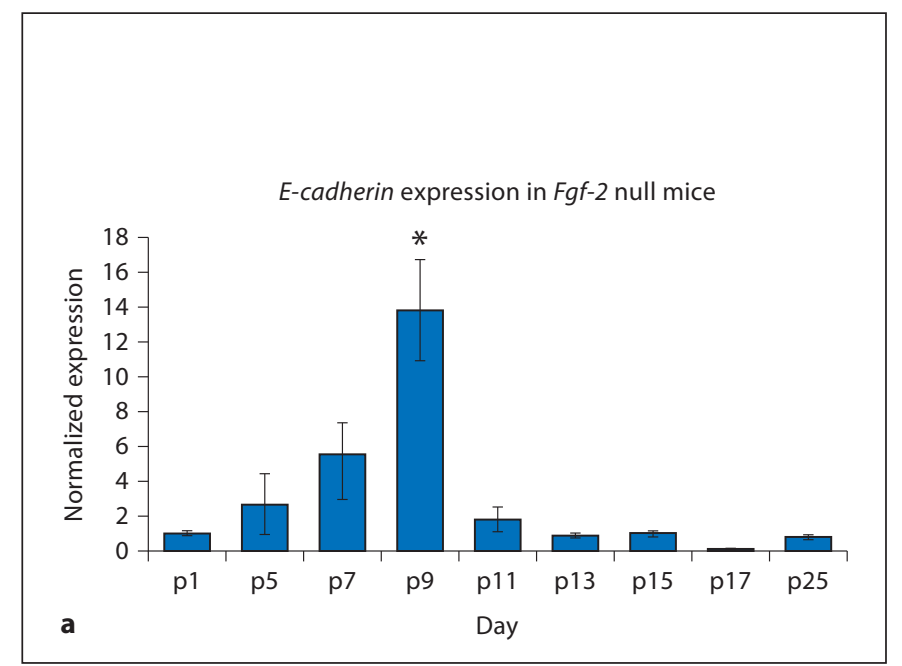

Fig. 5. E-cadherin expression is not down-regulated in the PF suture of $F g f-2$ null mice. a E-cadherin expression in PF suture of $\mathrm{Fgf}-2$ null mice during the first month of life was assessed by quantitative real-time PCR. Up-regulation of E-cadherin gene is observed starting on $\mathrm{p} 5$ with maximal level on $\mathrm{p} 9$ and return to baseline by p11. Note that up-regulation of E-cadherin gene in Fgf-

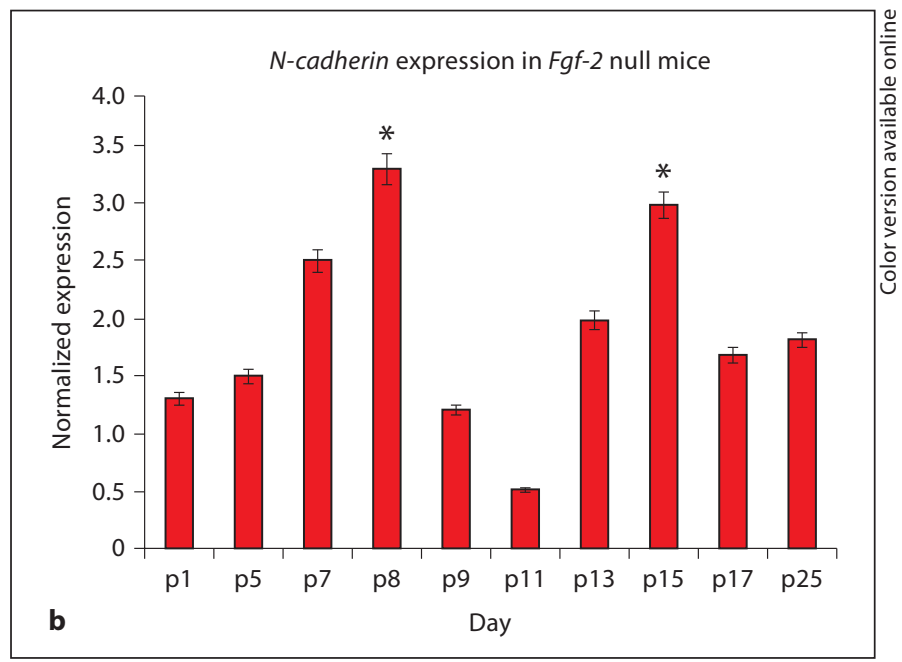

2 null mice occurs earlier than in CD-1 wild-type mice. b $\mathrm{N}$-cadherin expression in PF suture of Fgf-2 null mice also shows a pattern similar to that of wild-type CD-1 mice. Quantified mRNA values were normalized by the amounts of Gapdh mRNA, and results are given as fold induction. The results are presented as the mean \pm SD of 3 independent experiments. ${ }^{*} \mathrm{p}<0.05$.
Fig. 6. Temporal expression of $N$ - and $E$ cadherins and their regulators, Wnt7a and Snail. The diagram summarizes the temporal gene expression profiles and the key differentiation events occurring during PF suture closing.

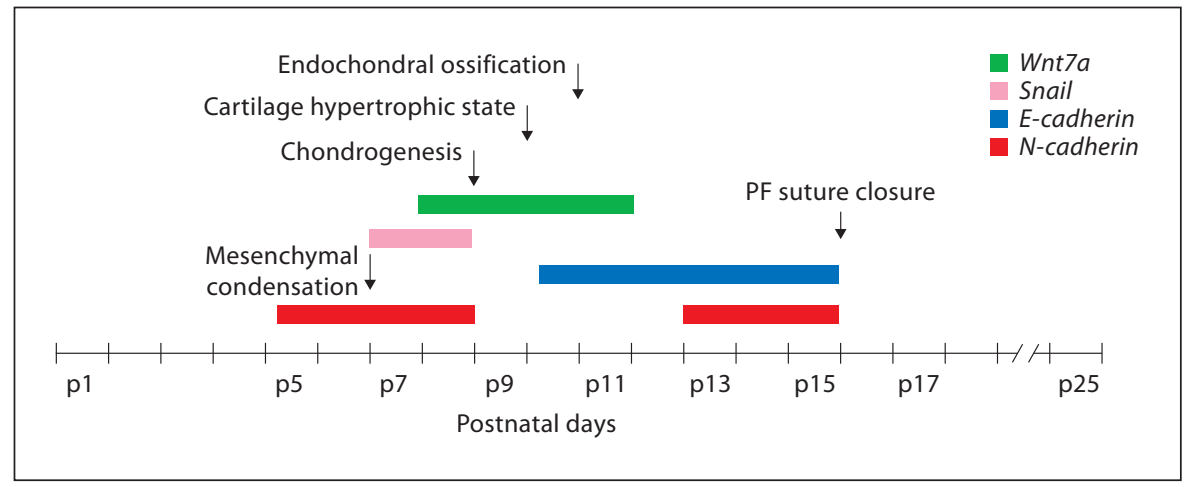

The PF suture closure occurs through endochondral ossification, and interestingly our study reveals a peculiar expression pattern of $\mathrm{N}$-cadherin during this process characterized by a biphasic up-regulation of this gene. A first up-regulation of $\mathrm{N}$-cadherin gene expression is observed at early time points when mesenchymal condensation occurs (pre-chondrogenesis) followed by a second up-regulation at late time points when suture closure is completed.

It is known that $\mathrm{N}$-cadherin down-regulation in precartilage condensation is a prerequisite step for mesen- chymal cells to differentiate into chondrocytes. Therefore, the precise modulation of $\mathrm{N}$-cadherin gene expression observed during the PF suture closure represents a crucial and permissive condition for the occurrence of suture closure. Indeed, our $\mathrm{N}$-cadherin expression results are fully in agreement with previous observations.

Studies suggest that a cross-talk between $\mathrm{N}$-cadherin and Wnt7a signaling occurs during the regulation of limb mesenchymal condensation. It has been demonstrated that misexpression of Wnt7a inhibits chondrogenesis in vitro, whereas Wnt5 a ligand activating the non-canoni- 
cal Wnt signaling does not [Tufan and Tuan, 2001]. Moreover, Wnt7a mis-expression influences the expression and/or turnover of $\mathrm{N}$-cadherin, thereby preventing it from being down-regulated at transition from condensation to chondrogenic differentiation. Interestingly, during PF suture closure we found a modulated expression of Wnt7 gene characterized by an up-regulation inversely correlating with that of $\mathrm{N}$-cadherin. These results suggest that Wnt7a is a potential negative regulator of $\mathrm{N}$-cadherin function during the chondrogenic differentiation occurring in the closing PF suture.

The late up-regulation of E-cadherin gene during the $\mathrm{PF}$ closure timing would suggest a potential role for this molecule during the final step, when osteogenesis takes place. Of interest, a previous study performed on OBcadherin null mutant mice showed a reduced calcified area at the PF suture that caused a round-shaped calvaria with increasing animal age to 3 months [Kawaguchi et al., 2001]. Indeed, this observation strongly supports the potential role of cadherins in the patterning and closure of PF suture.

Several studies indicated that E-cadherin gene expression is regulated by Snail, a transcriptional repressor which by binding to $3 \mathrm{E}$-boxes present in the E-cadherin promoter represses its transcription [Batlle et al., 2000]. Our analysis revealed in the closing PF suture at early time points an up-regulation of Snail gene overlapping with the down-regulation of E-cadherin expression. In contrast, at late time points Snail expression was downregulated concomitantly with the up-regulation of $E$ - cadherin expression. These results would suggest that $E$ cadherin expression in PF closing suture might be under the control of the transcriptional repressor Snail.

It is noteworthy that cadherins, also can modulate the canonical Wnt signaling. $\mathrm{N}$-cadherin and E-cadherin can interact directly with $\beta$-catenin and, thereby, may decrease Wnt signaling [Linask et al., 1997; Xu et al., 2002; Nelson and Nusse, 2004]. Moreover, a novel mechanism by which $\mathrm{N}$-cadherin interacts with the Wnt co-receptor LRP5 and regulates canonical Wnt/ $\beta$-catenin signaling in osteoblasts has been described [Hay et al., 2009].

Taken together, our results demonstrate that during the patterning and closure of the PF suture there is a precise and unique modulation of the 2 major adhesion molecules $\mathrm{N}$ - and E-cadherin. We believe that this modulation may represent timing of signaling events governing the patterning and closure of PF suture. Our current results and ongoing experiments would suggest that $\mathrm{E}$ - and $\mathrm{N}$-cadherins may represent a potential control of canonical Wnt signaling in PF suture. Indeed, the expression study presented herein sets the stage for future investigations aimed to further elucidate the molecular signaling underlying the patterning and closure of PF suture.

\section{Acknowledgments}

This work was supported by NIH grants R01DE13194 and R01 DE14526, and a grant from the Oak Foundation to M.T.L.

\section{References}

Batlle, E., E. Sancho, C. Franci, D. Dominguez, M. Monfar, J. Baulida, A. Garcia De Herreros (2000) The transcription factor snail is a repressor of E-cadherin gene expression in epithelial tumor cells. Nat Cell Biol 2: 84-89.

Bellus, G.A., K. Gaudenz, E.H. Zackai, L.A. Clarke, J. Szabo, C.A. Francomano, M. Muenke (1996) Identical mutations in three different fibroblast growth factor genes in autosomal dominant craniosynostosis syndromes. Nat Genet 14: 174-176.

Cano, A., M.A. Perez-Moreno, I. Rodrigo, A. Locascio, M.J. Blanco, M.G. del Barrio, F. Portillo, M.A. Nieto (2000) The transcription factor snail controls epithelial-mesenchymal transitions by repressing E-cadherin expression. Nat Cell Biol 2: 76-83.

Cho, S.H., C.D. Oh, S.J. Kim (2003) Retinoic acid inhibits chondrogenesis of mesenchymal cells by sustaining expression of $\mathrm{N}$-cadherin and its associated proteins. J Cell Biochem 89: 837-847.
Debiais, F., J. Lemonnier, E. Hay, P. Delannoy, J. Caversazio, P.J. Marie (2001) Fibroblast growth factor-2 (FGF-2) increases $\mathrm{N}$-cadherin expression through protein kinase $\mathrm{C}$ and Src-kinase pathways in human calvarial osteoblasts. J Cell Biocem 81: 68-81.

DeLise, A.M., R.S. Tuan (2002) Alterations in the spatiotemporal expression pattern and function of N-cadherin inhibit cellular condensation and chondrogenesis of limb mesenchymal cells in vitro. J Cell Biochem 87: 342-359.

-El-Hariry, I., M. Pignatelli, N.R. Lemoine (2001) FGF-1 and FGF-2 modulate the E-cadherin/ catenin system in pancreatic adenocarcinoma cell lines. Br J Cancer 15: 1656-1663.

Ferrari, S.L., K. Traianedes, M. Thorne, M.H. Lafage-Proust, P. Genever, M.G. Cecchini, V. Behar, A. Bisello, M. Chorev, M. Rosenblatt, L.J. Suva (2000) A role for N-cadherin in the development of the differentiated osteoblastic phenotype. J Bone Miner Res 15: 198-208.
Gumbiner, B.M. (1996) Cell adhesion: the molecular basis of tissue architecture and morphogenesis. Cell 84: 347-357.

Haas, A.R., R.S. Tuan (1999) Chondrogenic differentiation of murine c3H10T1/2 multipotent mesenchymal cells. II. Stimulation by bone morphogenetic protein-2 requires modulation of $\mathrm{N}$-cadherin expression and function. Differentiation 64: 77-89.

Hay, E., E. Laplantine, V. Geoffroy, M. Frain, T. Kohler, R. Muller, P.J. Marie (2009) N-cadherin interacts with axin and LRP5 to negatively regulate Wnt/beta-catenin signaling, osteoblast function, and bone formation. Mol Cell Biol 29: 953-964.

-Hajra, K.M., D.Y. Chen, E.R. Fearon (2002) The SLUG zinc-finger protein represses E-cadherin in breast cancer. Cancer Res 62: 16131618. 
Howard, T.D., W.A. Paznekas, E.D. Green, L.C. Chiang, N. Ma, R.I. Ortiz de Luna, C. Garcia Delgado, M. Gonzalez-Ramos, A.D. Kline, E.W. Jabs (1997) Mutations in TWIST, a basic helix-loop-helix transcription factor, in Saethre-Chotzen syndrome. Nat Genet 15: 36-41.

-Jamora, C., R. DasGupta, P. Kocieniewski, E. Fuchs (2003) Links between signal transduction, transcription and adhesion in epithelial bud development. Nature 422: 317-322.

-Jiang, X., S. Iseki, R.E. Maxson, H.M. Sucov, G. M. Morriss-Kay (2002) Tissue origins and interactions in the mammalian skull vault. Dev Biol 241: 106-116.

Kawaguchi, J., Y. Azuma, K. Hoshi, I. Kii, S. Takeshita, T. Ohta, H. Ozawa, M. Takeichi, O. Chisaka, A. Kudo (2001) Targeted disruption of cadherin-11 leads to a reduction in bone density in calvaria and long bone metaphyses. J Bone Miner Res 16: 1265-1271.

Kii, I., N. Amizuka, J. Shimonura, Y. Saga, A. Kudo (2004) Cell-cell interaction mediated by cadherin-11 directly regulates the differentiation of mesenchymal cells into the cells of the osteo-lineage and the chondro-lineage. J Bone Miner Res 19: 1940-1949.

- Larue, L., M. Ohsugi, J. Hirchenhain, R. Kemler (1994) E-cadherin null mutant embryos fail to form a trophectoderm epithelium. Proc Natl Acad Sci USA 91: 8263-8267.

Lemonnier, J., E. Hay, P. Delannoy, A. Lomri, D. Modrowski, J. Caverzasio, P.J. Marie (2001) Role of $\mathrm{N}$-cadherin and protein kinase $\mathrm{C}$ in osteoblast gene activation induced by the S252W fibroblast growth factor receptor 2 mutation in Apert craniosynostosis. J Bone Miner Res 16: 832-845.

Lemonnier, M., D. Modrowski, M. Hott, P. Delannoy, A. Lomri, P.J. Marie (1998) The Ser252Trp FGFR-2 mutation in Apert syndrome selectively increases E-cadherin and $\mathrm{N}$-cadherin expression in human calvaria osteoblasts in vitro and in vivo. J Bone Miner Res 23: S188.

-Linask, K.K., K.A. Knudsen, Y.H. Gui (1997) Ncadherin-catenin interaction: necessary component of cardiac cell compartmentalization during early vertebrate heart development. Dev Biol 185: 148-164.
Marie, P.J., F. Debiais, E. Hay (2002) Regulation of human cranial osteoblast phenotype by FGF-2, FGFR-2 and BMP-2 signaling. Histol Histopathol 17: 877-885.

Meyers, G.A., D. Day, R. Goldeberg, D.L. Daentl, K.A. Przylepa, L.J. Abrams, J.M. Graham Jr, M. Feingold, J.B. Moeschler, E. Rawnsley, A.F. Scott, E.W. Jabs (1996) FGFR2 exon IIIa and IIIc mutations in Crouzon, JacksonWeiss, and Pfeiffer syndromes: evidence for missense changes, insertions and a deletion due to alternative RNA splicing. Am J Hum Genet 58: 491-498.

Muenke, M., U. Schell, A. Hehr, N.H. Robin, H.W. Lossken, A. Schinzel, L.J. Pulleyn, P. Rutland, W. Reardon, S. Malcom, et al. (1994) A common mutation in the fibroblast growth factor 1 gene in Pfeiffer syndrome. Nat Genet $8: 269-274$.

Nelson, W.J., R. Nusse (2004) Convergence of Wnt, beta-catenin, and cadherin pathways. Science 303: 1483-1487.

Oberlender, S.A., R.S. Tuan (1994) Expression and functional involvement of $\mathrm{N}$-cadherin in embryonic limb chondrogenesis. Development 120: 177-187.

-Okazaki, M., S. Takeshita, S. Kawai, R. Kikuno, A. Tsujimura, A. Kudo, E. Amann (1994) Molecular cloning and characterization of OB-cadherin, a new member of cadherin family expressed in osteoblasts. J Biol Chem 269: 12092-12098.

Opperman, L.A. (2000) Cranial sutures as intramembranous bone growth sites. Dev Dyn 219: 472-485.

Renier, D., E. Lajeunie, E. Arnaud, D. Marchac (2000) Management of craniosynostoses. Childs Nerv Syst 16: 645-658.

Rice, D.P., R. Rice, I. Thesleff (2003) Molecular mechanisms in calvarial bone and suture development, and their relation to craniosynostosis. Eur J Orthod 25: 139-148.

-Sahar, D., M.T. Longaker, N. Quarto (2005) Sox9 neural crest determinant gene controls patterning and closure of the posterior frontal cranial suture. Dev Biol 280: 344-361.

Stains, J.P., R. Civitelli (2005) Cell-cell interactions in regulating osteogenesis and osteoblast function. Birth Defects Res 75: 72-80.
Strutz, F., M. Zeisberg, F.N. Ziyadeh, C.Q. Yang, R. Kalluri, G.A. Muller, E.G. Neilson (2002) Role of fibroblast growth factor-2 in epithelial-mesenchymal transformation. Kidney Int 61: 1714-1728.

$>$ Sood, S., Z.A. Eldadah, W.L. Krause, I. McIntosh, H.C. Dietz (1996) Mutation in fibrillin1 and Marfanoid-craniosynostosis (Shprintzen-Goldberg) syndrome. Nat Genet 12: 209-211.

Tuan, R.S. (2003) Cellular signaling in developmental chondrogenesis: N-cadherin, Wnts and BMP-2. J Bone Joint Surg 85: 137-141.

Tufan, A.C., K.M. Daumer, A.M. DeLise, R.S. Tuan (2002) AP-1 transcription factor complex is a target of signals from both Wnt-7a and $\mathrm{N}$-cadherin-dependent cell-cell adhesion complex during the regulation of limb mesenchymal chondrogenesis. Exp Cell Res 273: 197-203.

Tufan, A.C., R.S. Tuan (2001) Wnt regulation of limb mesenchymal chondrogenesis is accompanied by altered $\mathrm{N}$-cadherin-related functions. FASEB J 15: 1436-1438.

Tuli, R., S. Tuli, S. Nandi, X. Huang, P.A. Manner, W.J. Hozack, K.G. Danielson, D.J. Hall, R.S. Tuan (2003) Transforming growth factor-beta-mediated chondrogenesis of human mesenchymal progenitor cells involves $\mathrm{N}$ cadherin and mitogen-activated protein $\mathrm{ki}-$ nase and Wnt signaling cross-talk. J Biol Chem 278: 41227-41236.

Vleminckx, K., R. Kemler (1999) Cadherins and tissue formation: integrating adhesion and signaling. Bioessays 21: 211-220.

Wilkie, A.O. (1997) Craniosynostosis: genes and mechanisms. Hum Mol Genet 6: 1647-1656.

Wilkie, A.O., G.M. Morriss-Kay (2001) Genetics of craniofacial development and malformation. Nat Rev Genet 2: 458-468.

Xu, G., C. Arregui, J. Lilien, J. Balsamo (2002) PTP1B modulates the association of betacatenin with $\mathrm{N}$-cadherin through binding to an adjacent and partially overlapping target site. J Biol Chem 277: 49989-49997.

Yagi, T., M. Takeichi (2000) Cadherin superfamily genes: functions, genomic organization, and neurologic diversity. Genes Dev 14: 1169-1180. 